\title{
Superluminal Neutrinos: Experimental Data and New Interpretative Theories
}

\author{
*Luca Nanni \\ Università degli Studi di Ferrara, G. Saragat 1, 44100-Ferrara (Italy) \\ *e-mail of corresponding author: luca.nanni@student.unife.com
}

\begin{abstract}
In this study the data of the OPERA and MINOS experiments, together with those related to the SN1987A supernova, are discussed in the context of the recent theories proposed for the superluminal muon neutrino. It is proved that for the models in which the Lorentz symmetry is violated, the decay mechanism leading to neutrino oscillation becomes possible. Within this framework, a new model based on the Hartman effect is proposed, according to which the neutrino becomes superluminal by quantum tunnelling, crossing a potential barrier generated by its interaction with the earth's crust matter. This model does not violate the Lorentz symmetry since the tachyonic state is generated by the quantum fluctuation of the neutrino initial energy, even if it requires to conjecture the presence of a quantum field that we ascribe to be that due to dark matter. In this model all superluminal neutrino decay mechanisms proposed in other studies are allowed. The hypothetical boson mediating the interaction between neutrino and dark matter is also discussed.
\end{abstract}

Pacs: $13.15 .+\mathrm{g}, 14.60 . \mathrm{Pq}, 12.60 .-\mathrm{i}, 14.80 .-\mathrm{j}$

Keywords: tachyons; neutrino; superluminal tunneling; neutrino oscillation; dark matter

\section{Introduction}

Neutrinos play a key role in particle and nuclear physics as well as in astrophysics [1-4]. Postulated by Pauli in 1930, they were experimentally discovered by Reines and Cowan in 1956 [5]. A second family of neutrinos was discovered in 1962 and a third in 1975 [6-7]. The CERN collider LEP proved in 1989 that three types of interacting neutrinos, named electron, muon and tau neutrino, are enough in the standard model of particle physics [8-9]. They are uncharged particles with a very tiny mass and interact only weakly, so to cross large quantity of matter without interacting. For this reason neutrinos are considered particles that could contain information from the early universe (relic neutrinos) [10-12]. Neutrinos are emitted in huge numbers in nuclear reactors and are artificially produced in modern accelerators. However, the main source of neutrinos remains the Universe, with its infinity of cosmological processes (stellar nucleosynthesis, supernovae, powerful binary systems, microquasars, active galactic nuclei, gamma ray bursts and Big Bang) [13-15]. 
However, although neutrino is among the most abundant particles in nature and although physics has been devoting to it for almost a century of greatest efforts, both theoretical and experimental, paradoxically it remains the least known and bizarre within the Standard Model. The neutrino mass origin, its flavor oscillations, the existence of Majorana neutrino (a particle which is also its own antiparticle), the sterile neutrino, are all questions to which physics still fails to respond with a coherent and complete theory of particles [16-19]. To this picture we must also add the superluminal neutrino, with its theories and experimental evidences considered by many to be speculative and misinterpreted but that could threaten part of the orthodoxy of the actual Standard Model [20-24]. It is precisely on this last point that the attention of this article will be focused, whose purpose is to review the recent experimental results and the proposed interpretative theories. Then, experimental data will be interpreted with a new theory, based on superluminal tunneling $[25,26]$, which correlates the superluminal behaviour of neutrino with its flavor oscillation and that indirectly predicts the existence of dark matter. All experimental data currently available, relating to the OPERA [27] and MINOS [28] experiments and to measurements of neutrino and photon beams from the Supernova SN1987A [29], are considered by most of the scientific community as anomalies, some of which due to apparatus malfunctions. In this study we want to start from a different perspective, considering that even if most of the scientific community is sceptical [30], in any case there are not proves that neutrino superluminality is forbidden a priori. The existence of the positron, the idea of the neutrino, the spacetime curvature due to mass, are examples for which the theory has anticipated (and probably guided) experimental discoveries. For this reason, we believe that it is worth continuing the theoretical study on superluminal motions, especially for those particles that, like neutrino, weakly interact with ordinary matter and that can reach energy values at the upper limit allowed by the current technology. This could facilitate the design of new experiments to investigate the neutrino oscillation and its possible superluminal states.

\section{Experimental Data}

The experimental data considered in this study are those relating to OPERA and MINOS experiments and to the detection of neutrinos from the Supernova SN1987A [27-29]. In Table 1 are listed the numerical values of physical quantities that will be used in the following: 


\begin{tabular}{|c|c|c|c|c|}
\hline & Beam Energy & Baseline & $\delta \mathbf{t}$ & Neutrino Velocity \\
\hline & $\mathrm{GeV}$ & $\mathrm{Km}$ & ns & $\mathrm{Km} / \mathrm{s}$ \\
\hline \multirow{4}{*}{ OPERA } & 13.8 & \multirow{4}{*}{$730.53461 \pm 2 \cdot 10^{-4}$} & $-54.7 \pm 18.4[\text { stat. }]_{-6.9}^{+7.3}[$ sys. $]$ & $306908.628 \pm 2$ \\
\hline & 17.0 & & $-57.8 \pm 7.8[\text { stat. }]_{-5.9}^{+8.3}[$ sys. $]$ & $307308.855 \pm 1$ \\
\hline & 28.2 & & $-61.1 \pm 13.0[\text { stat. }]_{-6.9}^{+7.3}[$ sys. $]$ & $307736.050 \pm 2$ \\
\hline & 40.7 & & $-68.1 \pm 19.1[\text { stat. }]_{-6.9}^{+7.3}[$ sys. $]$ & $308646.146 \pm 3$ \\
\hline MINOS & 3.0 & $734.2986 \pm 0.7$ & $-126 \pm 32[$ stat. $] \pm 64[$ sys. $]$ & $299830.232 \pm 5$ \\
\hline SN1987A & --- & $1.58605 \cdot 10^{18}$ & $-10,800(\mathrm{~s})$ & 299820.416 \\
\hline
\end{tabular}

Table 1

where $\delta \mathrm{t}$ is the anticipation time, calculated considering the difference between the measured neutrino velocity and the speed of light.

According to Standard Model, neutrino interacts with ordinary matter, formed by baryons and leptons, through a charged current mechanism (CC) mediated by $\mathrm{W}$ boson, and a neutral current mechanism (NC) mediated by $\mathrm{Z}$ boson [31]. In the OPERA and MINOS experiments neutrino beam travelled through a layer of matter made of aluminium-silicates (earth crust) and magnesium-silicates (terrestrial mantle). Using the geological data relating to the first kilometres of earth depth [32] and a bit of stoichiometry, the results listed in Table 2 are easily obtained:

\begin{tabular}{|c|c|c|c|c|c|c|c|}
\hline $\mathbf{S i O}_{2}$ in crust & $\mathbf{S i O}_{2}$ in mantle & $\mathbf{A l}_{\mathbf{2}} \boldsymbol{O}_{\mathbf{2}}$ in crust & $\mathbf{M g O}$ in mantle & $\boldsymbol{\rho}_{\text {crust }}$ & $\boldsymbol{\rho}_{\text {mantle }}$ & Total Baryons & Total Leptons \\
\hline$\%$ & $\%$ & $\%$ & $\%$ & $\mathrm{gr} / \mathrm{cm}^{3}$ & $\mathrm{gr} / \mathrm{cm}^{3}$ & $\mathrm{~N}^{\circ} / \mathrm{cm}^{3}$ & $\mathrm{~N}^{\circ} / \mathrm{cm}^{3}$ \\
\hline 60 & 46 & 15 & 39 & 2.7 & 3.3 & $1.8 \cdot 10^{24}$ & $9.0 \cdot 10^{23}$ \\
\hline
\end{tabular}

Table 2

The total number of baryons has been calculated as the number of nucleons present in the most abundant and stable isotopes of the atoms forming the earth's matter, while the total leptons is the number of electrons in the inorganic molecules. Considering the energies involved in the OPERA and MINOS experiments, the total neutrino cross section $\sigma_{\text {tot }}$ is of the order of $10^{-38} \mathrm{~cm}^{2} / \mathrm{GeV}$ [33]. The scattering probability per unit of length of neutrino with the matter is [34]:

$$
\frac{d P}{d x}=\sigma_{t o t} \rho
$$

where $\rho$ is the number of baryonic or leptonic particles per cubic centimetre. Substituting in Eq. 1 the respective numerical values we obtain:

$$
\left(\frac{d P}{d x}\right)_{\text {baryonic }} \cong\left(\frac{d P}{d x}\right)_{\text {leptonic }} \cong 10^{-14} \text { events } / \mathrm{cm} \cdot \mathrm{GeV}
$$

Eq. 2 is the probability that a neutrino with energy of $1 \mathrm{GeV}$ interacts with a baryonic or leptonic particle covering a distance of $1 \mathrm{~cm}$. By Eq. 1 we calculate also the average distance that neutrino may travel freely before that an interaction occurs:

$$
\lambda=\frac{1}{\sigma_{\text {tot }} \rho} \cong 10^{12} \mathrm{~m} \cdot \mathrm{GeV} / \text { particle }
$$


Eq. 3 gives a better idea why a single neutrino has a very low probability to interact with earth matter. In the quantum tunneling theory this is a fundamental point to explain why superluminal neutrino states are very rare and become considerable only when they travel with high energy through high density matter.

In the case of the supernova SN1987A, the neutrino crosses cosmic matter, mainly formed by hydrogen, with an average density of $10^{-2}$ particles $/ \mathrm{cm}^{3}$ [35]. The calculated energy of neutrinos produced from a supernova of the type SN1987A is up to $30 \mathrm{MeV}$ [36], which corresponds to a cross section of $10^{-40} \mathrm{~cm}^{2} / \mathrm{GeV}$. By these data the calculated average distance $\lambda_{S N 1987 S}$ that neutrinos can travel without interact with cosmic matter is:

$$
\lambda_{S N 1987 \mathrm{~A}}=\frac{1}{\sigma_{30 \mathrm{MeV}} \rho_{\text {cosmic }}} \cong 10^{40} \mathrm{~m} \cdot \mathrm{GeV} / \text { particle }
$$

Therefore, neutrino beams from supernovae can propagate in cosmic space practically unperturbed. The ratio between the baseline and $\lambda$ gives the number of neutrino-matter interaction:

$$
\left\{\begin{array}{l}
\frac{L_{O P E R A}}{\lambda_{\text {OPERA }}}=7.3 \cdot 10^{-7} \text { events } / \mathrm{GeV} \\
\frac{L_{M I N O S}}{\lambda_{M I N O S}}=7.3 \cdot 10^{-7} \text { events } / \mathrm{GeV} \\
\frac{L_{S N 1987 A}}{\lambda_{S N 1987 A}}=1.6 \cdot 10^{-19} \text { events } / \mathrm{GeV}
\end{array}\right.
$$

These results will be used in section 4 dedicated to the new interpretative theory that is proposed and discussed in this research work.

\section{Current Interpretative Theories}

The superluminal neutrino reported by OPERA, even if denied by the scientific community, has awakened interest in research concerning the violation of Lorentz symmetry [37]. Recently, many new interpretative theories have been proposed, aimed to explaining the results of OPERA as well as those concerning the MINOS collaboration and those concerning the neutrino beam from the SN1987A supernova. These models deviate, more or less speculatively, from the Standard Model which in its formalism is strictly faithful to the theory of special relativity. In this section we want to review the most relevant interpretative models of authoritative researchers with recognized experience in the field of neutrino physics or in theoretical physics.

In this perspective, one of the most cited models in the bibliography is that of Cohen and Glashow (CG) [38]. This model assumes that in superluminal motions some kinematic processes, otherwise forbidden, become possible without violating the impulse and energy 
conservation laws. In this model the superluminal neutrino is an unstable particle that decays following a mechanism like that of Cherenkov:

$$
\left\{\begin{aligned}
\left(v_{\mu}\right)^{\text {Superluminal }} & \rightarrow v_{\mu}+\gamma \\
\left(v_{\mu}\right)^{\text {Superluminal }} & \rightarrow v_{\mu}+v_{e}+\bar{v}_{e} \\
\left(v_{\mu}\right)^{\text {Superluminal }} & \rightarrow v_{\mu}+e^{-}+e^{+}
\end{aligned}\right.
$$

The first and third processes are kinematically allowed, while the second is forbidden because neutrinos with the same energy and different flavour travel at the same velocity. The OPERA experiment did not detect any photon or pair emissions; however, it must be considered that the superluminal neutrino loses energy while it propagates in the earth's crust and therefore these phenomena could not be detected in the Gran Sasso laboratories. So, the CG model remains, from a theoretical point of view, a valid interpretative theory applicable also to the other experimental results that show a superluminal neutrino behaviour. The difficulty of this model lies in the fact that it starts from the assumption that the neutrino is superluminal, but it says nothing about how the neutrino can be born as a tachyon or how it can become superluminal while traveling within the matter.

The same CG approach was also followed by Jentschura [39], but in this case it is conjectured that at high energy values (at least in the order of $\mathrm{GeV}$ ) the neutrino interacts with a (unknown) field which gives it superluminal properties and a mass that depends on its enery. This conjecture, speculative but needed if we want to give an explanation to anomalous experimental data, makes the second mechanism of Eq. 6 possible. In fact, since the neutrino mass is energy dependent, the electron neutrino-antineutrino velocity cannot be equal to that of the muon neutrino. In other words, the decay mechanism $\left(v_{\mu}\right)^{s} \rightarrow v_{\mu}+v_{e}+\bar{v}_{e}$ is possible provided that at high energies the kinematic parameters of the different neutrino mass eigenstates differ from each other. The Jentschura model will be taken up in section 4 of this article, where the formulation of a new interpretative theory of the superluminal neutrino is given.

Another original theory [40] is inspired to the Majorana equation for particles with arbitrary spin [41]. This equation, formulated to be invariant respect the infinitesimal transformations of the Lorentz group, admits tachyonic solutions. The bradyonic neutrino, traveling through the earth's crust, acquires an imaginary mass remaining a stable particle. In fact, the mass-energy dispersion relationship of a tachyon, given by $E^{2}=p^{2} c^{2}-m^{2} c^{4}$, forbids the formation of couples for which the condition $E^{2}-p^{2} c^{2}>\left(m c^{2}\right)^{2}$ must be satisfied. Within the Standard Model this behaviour can be reproduced only by modifying the 
usual energy-moment dispersion relationship, more precisely by introducing a term $F$ that makes it non-linear:

$$
E^{2}=p^{2} c^{2}+m^{2} c^{4}+F
$$

The arbitrary function $F$ suppresses the pair production when assumes negative value [42]. If we match Eq. 7 with the tachyonic mass-energy dispersion relationship, the value of $\mathrm{F}$ becomes $F=-2\left(m c^{2}\right)^{2}$, which correspond to the negative value of the threshold energy for a pair production. As a matter of fact, the term $F$ leads to a weak space-time Lorentz symmetry breaking. In this theory the authors conjecture that the neutrino produced at LHC becomes superluminal due to the interaction with the hypothetical sterile neutrino within the gravitational field. In this sense this model is like that proposed by Jentschura (in which the neutrino interacts with an unknown field), but with the difference that the obtained tachyon is stable and does not lose energy due to the Cherenkov effect. Therefore, in this case the detector of the Gran Sasso Labs detected a tachyon, while according to the other mentioned theories it detected a muon neutrino which during part of its journey travelled at superluminal speed.

Regarding neutrinos from the SN1987A supernova, their superluminality has been discussed implicitly in Huzita work [43]. The author never mentions the tachyonic neutrino, on the contrary he calculates the arrival time and its dispersion considering that the beam is formed by neutrinos with different mass eigenstates, trying to discern the disagreement between experimental and theoretical data by playing on the values of such states. In other words, he tries to fit the experimental data with the Standard Model theory, using however a conjecture as speculative as that of the superluminal neutrino. Furthermore, Huzita assumes that the formation of different mass states occurs through the interaction of neutrinos with the dense stellar matter in the initial phases of the supernova explosion, remaining unchanged on their journey through empty space. All these features are in fact those typical of superluminal neutrino behavior.

\section{$4 \quad$ Superluminal Neutrino through Tunnelling}

In this section the experimental data listed in Table 1 are interpreted by a theory based on the Hartmann effect that characterizes the quantum tunnelling of particles through a wide potential barriers [44]. Recently this phenomenon has also been extended to spin-1/2 particles [45-46]. Quantum tunnelling is governed by the uncertainty principle contemplated by the Standard Model. In this model it is conjectured that the potential barrier $U$ is generated by the interaction of the neutrino with the matter through $\mathrm{CC}$ mechanisms, mediated by $\mathrm{W}$ bosons, 
and $\mathrm{NC}$, mediated by $\mathrm{Z}$ bosons. In other words, these interactions are equivalent to a sort of potential barrier that counteract the free propagation of neutrino. As can be seen we are still proceeding within the formalism of the Standard model without the need to introduce other speculative hypotheses.

Within the potential barrier neutrino becomes an evanescent wave and, for enough large barrier, it behaves like a superluminal particle, being the tunnelling time independent from the barrier geometry [47]. In our case the barrier height is proportional to the number of neutrino interactions with the matter it crosses, and its width depends on the maintenance of these interactions along the baseline. The tachyonic energy is therefore given by:

$$
E_{\text {tach. }}=U-E=\gamma_{\text {tach. }} m c^{2}
$$

where $\gamma_{\text {tachyonic }}$ is the tachyonic Lorentz factor. Solving Eq. 8 for tachyon velocity $u_{\text {tachyonic }}$ we get:

$$
u_{\text {tach. }}=c\left(1+\frac{m^{2} c^{4}}{(U-E)^{2}}\right)^{1 / 2}
$$

Eq. 9 agrees with the provisions of the Jentschura and Laveder-Tamburini models, according to which the tachyonic velocity of neutrino depends on its mass and initial energy. The tachyon state inside the barrier is very unstable and its mean lifetime is given by the uncertainty principle:

$$
\delta t=\hbar / E
$$

Thus, the violation of Lorentz space-time symmetry is nothing but a typical quantum fluctuation. Plotting the tachyon velocities vs the respective energies (see Table 1) the following trend is obtained:

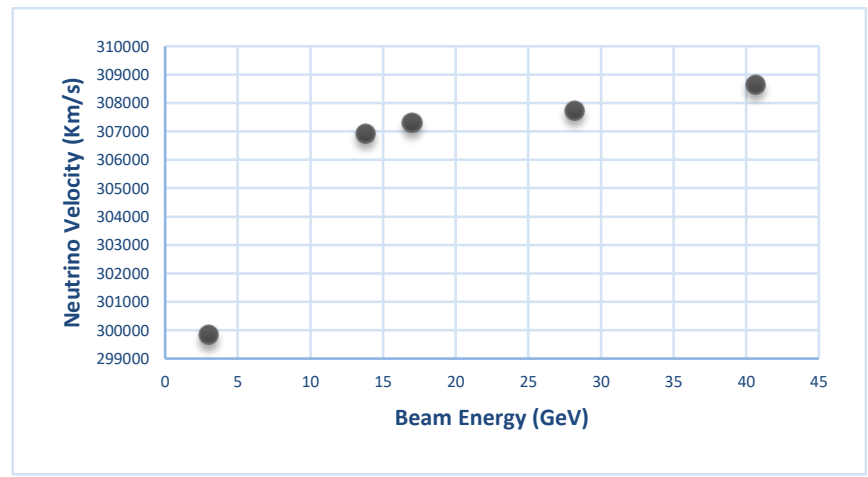

Figure 1: Neutrino velocity vs neutrino energy

The best fitting returns the following equation:

where:

$$
u_{\text {tach. }}=a E^{b}
$$

$$
\left\{\begin{array}{c}
a=296,858.900 \pm 1,522.000 \mathrm{Km} / \mathrm{s} \\
b=0.01123321 \pm 0.001781 \\
R^{2}=0.9306 ; \quad p-\text { value }(\alpha=5 \%)=0.01828
\end{array}\right.
$$


The quantity $a$ is the $99 \%$ of the speed of light and considering the error affecting it we can say that is very close to $c$. However, for consistency of the units of measure it will be needed to have:

$$
a=c / H^{b}
$$

where $H$ is an energy value such that $(E / H)^{b}>1$. Eq. 10 then becomes:

$$
u_{\text {tach. }}=c(E / H)^{b}
$$

Using Eqs. 12 and 13, the value of $H$ is easily calculated:

$$
H=(c / a)^{1 / b}=2.3998 \mathrm{GeV}
$$

To understand the physical meaning of $H$ we calculate, by means of Eq. 11, the energy of the neutrinos coming from the Supernova SN1987A:

$$
E_{S N 1987 A}=2.42 \mathrm{GeV}
$$

We also observe that the velocity detected for these neutrinos is only 1,00037 times higher than that of light, consistent with the relatively small value of $E_{S N 1987 A}$. Therefore, we can assume that $H$ is the minimum energy value, due to the interaction of neutrino with the matter, which makes possible in probabilistic terms the transition to the superluminal state. In other words, $H$ is a threshold energy and the minimum height of the potential barrier depends on the energy value with which the initial beam is produced:

$$
U_{\min }=E+H
$$

Since the transition to the tachyonic state can occur only if $(E / H)>1$, it follows that the initial energy of the muon neutrino must be not less than $2.3998 \mathrm{GeV}$. Furthermore, according to our model, the tunnelling neutrino must at least double this energy by means of CC and NC interactions with the nucleons of the matter. Using Eq. 2, the average number of interaction events of $\mathrm{a} \approx 2.4 \mathrm{GeV}$ neutrino travelling through $\mathrm{a} \approx 730 \mathrm{Km}$ baseline with matter is about $1.75 \cdot 10^{-6}$, while for the SN1987A neutrino this value decreases to $3.84 \cdot 10^{-19}$. Supposing that neutrino-matter interaction energy is of the order of the $\mathrm{MeV}$, to have a potential barrier equal to $E+H$ it is necessary that the effective interaction events are at least of the order of $10^{3}$. Therefore, it is not reasonable to ascribe the potential barrier to the sole interaction of neutrino with ordinary matter, but it is necessary to introduce the hypothesis that during his journey the neutrino interacts with other matter (dark matter?) with very high density or that interacts very strongly with neutrino. This is the first and only conjecture that we are forced to introduce in our interpretative model to keep it consistent with experimental data. This conjecture, however, does not represent a new speculation since the link between neutrino physics and dark matter is a topic discussed and studied for decades in the field of particle physics [48-49].

Let's return to the function of best fitting of Eq. 11; equating it to the Eq. 9 we get:

$$
(E / H)^{b}=u_{\text {tach. }} / c=\beta_{\text {tach }}
$$


and the tachyonic energy given by Eq. 8 becomes:

$$
E_{\text {tach. }}=\frac{1}{\sqrt{(E / H)^{2 b}-1}} m c^{2}
$$

However, the model we are studying does not provide the tachyonic energy inside the potential barrier and, consequently, does not allow to obtain the value of the imaginary neutrino mass. Nevertheless, we can suppose that the superluminal state has a mean lifetime lower than the anticipation time $\delta$ t. In other words, within the barrier the probability that the neutrino occupies a tachyon state is limited to a fraction of the baseline. So, invoking the uncertainty principle, whose formalism is also valid for tachyons [50], the tachyonic energy is:

$$
E_{\text {tach. }} \delta t \geq \hbar \quad \rightarrow \quad E_{\text {tach. }} \geq 10^{-7} \mathrm{eV}
$$

where the uncertainty on the time is of the order of $n s$. Considering that the tachyonic Lorentz factors corresponding to the velocities listed in Table 1 are of the order of units or tens of units, the modulus of the tachyonic neutrino mass is $m_{\text {tach. }} \geq 10^{-8} \mathrm{eV}$. This is a very small but expected value since, as anticipated above, the superluminal state is very unstable. This result find also confirmation in Albrow's work on tachyonic dark matter [51].

\section{Discussion}

The idea of interpreting the superluminal neutrino using the Hartman effect is to be considered original, given that to the best of our knowledge in the literature there are no articles published on the subject. Since the tunnelling is governed by the uncertainty principle, the superluminal state obtained is highly unstable and has a mean lifetime which increases with increasing the energy of the initial bradyonic neutrinos. In fact, using Eqs. 10 and 19 we obtain:

$$
\delta t=\hbar \frac{\sqrt{(E / H)^{2 b}-1}}{m c^{2}}
$$

This model, therefore, explains the superluminal behaviour of neutrino as a quantum fluctuation, avoiding in a simple way problems and speculations inherent the violation of the Lorentz symmetry that affect the theory discussed in the previous section. Nevertheless, it requires to introduce the hypothesis of a new quantum field that interacts with neutrino through a force much more intense than that characterizing the interaction mechanisms with ordinary matter. Without going out of the schemes of current theoretical research, we can admit that this new quantum field is due to dark matter, whose existence is proved indirectly by cosmological and astronomical studies on galaxies [52]. In this way we can explain why 
superluminal neutrino remains a rare phenomenon confined to high-energy physics (higher than the $\mathrm{GeV}$ ), but still detectable in experiments such as OPERA and MINOS that have made use of very small baselines and matter (the terrestrial one) of low density.

Eq. 21 proves that the mean lifetime of tachyonic neutrino is closely connected with the energy of the initial beam; more precisely, the greater the energy of the neutrino produced and the more stable is its tachyonic form. This result is consistent with what is expected from the dynamics of tachyons, where the energy decreases as its superluminal velocity increases [53]. All the obtained equations, however, do not allow to calculate separately the tachyonic energy inside the potential barrier and the neutrino imaginary mass. The only prediction we can make about the absolute value of the imaginary mass, with the help of the uncertainty principle, is to calculate its lower limit and infer that the corresponding quantum state is highly unstable. The value of this limit, however, is completely different from that obtained in reference [42] $(0.15 \mathrm{eV})$ and from that calculated by Ehrlich in reference [54] $(0.63 \mathrm{eV})$. This difference is justified by the fact that in the model we are proposing the tachyon is a very unstable quantum state while the other theories are based on models that violate Lorentz symmetry and predict that tachyon is a stable particle.

The value of the threshold energy $H$ remains to be discussed; it can be thought as a kind of activation energy to pass from a bradyonic to a tachyonic state. So, the value of $2.3998 \mathrm{GeV}$ could be that of the boson mediating the interaction between the neutrino and the dark matter. The mean lifetime of this boson is:

$$
\delta t \geq \hbar / H \quad \rightarrow \quad \delta t \geq 2,710^{-25} \mathrm{~s}
$$

The number of interactions between neutrino and dark matter along the baseline is easily calculated as ratio between the anticipation time $\delta t$ and mean lifetime of Eq. 22. In MINOS experiment, for instance, the number of interactions is of the order of $10^{21}$; by this value we get the mean interaction length:

$$
\lambda_{\text {inter. }}=L_{\text {MINOS }} / n^{\circ} \text { interactions } \rightarrow \lambda_{\text {inter. }} \cong 7,3410^{-16} \mathrm{~m}
$$

This length is typical of the atomic nucleus, which means that this force is of short range. Since the inverse of $\lambda_{\text {inter. }}$ gives the term $\sigma \rho$, we can infer that the cross section of this new interaction is very high, of the order of Tera or Peta $\mathrm{cm}^{2} / \mathrm{GeV}$.

We conclude this section by pointing out that the tunnelling model is much closer to that proposed by Jentschura [39] and discussed in paragraph 3. Indeed, although the Lorentz symmetry is not violated, the module of the tachyonic imaginary neutrino mass depends on the energy of the initial beam, as proved by Eq. 19. Consequently, the possibility that the 
tachyonic state decays according to the second mechanism of Eq. 6, in which flavour oscillation occurs, is plausible within this model.

\section{Conclusion}

The proposed model provides a detailed explanation of the experimental data relating to the OPERA and MINOS experiments and to neutrino anomaly from the SN1987A supernova. The Hartman effect theorises the existence of the superluminal state as a quantum energy fluctuation and explains why the superluminal neutrino travels at speeds slightly higher than that of light. This model suggests that superluminality and flavour oscillation are related phenomena and, indirectly, provides a further confirmation of the presence of dark matter. Further confirmation of its correctness may come from the next experiments, such as KATRIN [55] and IceCube observatory [56]. These experiments, in fact, have been designed to study neutrinos with energy of the order of $\mathrm{PeV}$ which, according to our model, are those that can lead to more stable tachyonic states, with a mean life such as to further reduce the experimental uncertainties affecting the anticipation time measurements.

\section{References}

1. V. Barger, D. Marfatia, K. Wishnant, The Physics of Neutrino, Princeton University Press, Princeton (2012).

2. J.W.F. Valle, Proceeding of Science, Neutrino Oscillation Workshop, Brindisi (2018).

3. S.T. Pectov, Advances in High Energy Physics, 852987 (2013).

4. R.N. Mohapatra et al., Reports on Progress in Physics, 70, 1757 (2003).

5. C.L. Cowan, F. Reines, F.B. Harrison, H.W. Kruse, A.D. McGuire, Science, 124, 103 (1956)

6. D.B. Cline, Comments on Nuclear and Particle Physics, 17, 145 (1987).

7. M. Chemarin, Nuclear Physics B-Proceedings Supplements, 85, 67 (2000).

8. S.M. Bilenky, Phys. Part. Nuclei, 46, 475 (2015).

9. H. Pas, Annalen Phys., 11,551 (2002).

10. B. Follin, L. Knox, Z. Pan, Phys. Rev. Lett., 115, 091301 (2015).

11. A.D. Dolgov, Phys. Rept., 370, 333 (2002).

12. A.D. Dolgov, Phys. Atom. Nucl., 712152 [0803.3887] (2008).

13. P. Meszaros, Annual Rev. Nucl. Part. Science, 67, 45 (2017).

14. C. Yanagisawa, Front. Phys., 10, 1 (2014).

15. T. Weiler, Astropisical. Journal, 285, 495 (1985).

16. H. Murayama, Progress in Particle and Nuclear Physics, 57, 3 (2006).

17. O.G. Miranda, J.W.F. Valle, Nuclear Physics B, 908, 436 (2016).

18. E. Recami, Hadronic Journal, 40, 149 (2017).

19. T. Lasserre, Physics of the Dark Universe, 4, 81 (2014).

20. J. Alexandre, J. Ellis, N.E. Mavromatos, Physics Letters B, 706, 456 (2012). 
21. R. Suleiman, Progress in Physics, 4, 53 (2013).

22. T. Adam et al., Journal of High Energy Physics, 10, 93 (2012).

23. M. Antonello et al., Journal of High Energy Physics, 2012:49 (2012).

24. R. Ehrlich, Astroparticle Physics, 85, 43 (2016).

25. E. Recami, Foundations of Physics, 31,1119 (2001).

26. V. S. Olkhovsky, E. Recami, G. Salesi, Europhysics Letters, 57, 879 (2002).

27. N. Agafonova et al., J. High Energ. Phys., 2012:93 (2012).

28. P. Adamson et al., Phys. Rev. D, 76:072005 (2007).

29. D. Fargion et al., arXiv:1109.5368.

30. A.G. Cohen, S.L. Glashow, Phys. Rev. Lett., 107, 181803 (2011).

31. F. Vannucci, Progress in Particle and Nuclear Physics 95, 1 (2017).

32. U.S. Geological Survey (USGS) Coastal and Marine Geology Program (CMGP) Pacific Coastal and Marine Science Center (PCMSC): Archive of data and information related to the USGS PCMSC Samples Repository geoscience collections. NOAA National Centers for Environmental Information. doi:10.7289/V55T3HGJ.

33. M. Tanabashi et al. (Particle Data Group), Phys. Rev. D 98, 030001 (2018).

34. C. Patrignani et al. (Particle Data Group), Chin. Phys. C, 40, 100001 (2016) and 2017 update.

35. T.W. Noonan, Pub. Astron. Soc. Pacific, 83, 31 (1971).

36. P. Galeotti, G. Pizzella, Physics of Atomic Nuclei, 81, 105 (2017).

37. A. Jenkins, Phys. Rev. D, 69105007 (2004).

38. A.G. Cohen, S.L. Glashow, Phys. Rev. Lett., 107181803 (2011).

39. U.D. Jentschura, Cent. Eur. J. Phys., 10,749 (2012).

40. M. Laveder, F. Tamburini, arXiv:1111.4441.

41. E. Majorana, Nuov. Cim., 9, 335 (1932).

42. M. Laveder, F. Tamburini, arXiv:1109.5445.

43. H. Huzita, arXiv:hep-ph/0212337.

44. T.E. Hartman, J. Appl. Phys., 33 (12), 3427 (1962).

45. L. Nanni, Int. J. Mod. Phys.: Conference Series, 47, 1860102 (2018).

46. L. Nanni, Preprints 2019040048 (2019).

47. H.G. Winful, Phys. Rep., 436 (1-2), 1 (2011).

48. S. Pandey, S. Karmakar, S.J. Rakshit, High Energ. Phys., 2019:95 (2019).

49. E. Roulet, Phys. Rev. D, 475247 (1993).

50. V.F Perepelista, arXiv:1407.3245[physics.gen-ph].

51. M. Albrow, Proceeding of Science, 2nd World Summit: Exploring the Dark Side of the Universe 25-29 June 2018. arXiv:1811.03470[physics.gen-ph].

52. L.M. Krauss, Dark matter in the universe. Scientific American 255 (6), 58 (1986).

53. R.L. Dawe, K.C. Hines, Austr. J. Phys., 45(6), 725 (1992).

54. R. Ehrlich, Advances in Astronomy, ID 2820492 (2019).

55. G.B. Franklin, arXiv:1809.10603v1 [physics.ins-det].

56. T. Kubavara et al., Proceeding of Science, $34^{\text {th }}$ International Cosmic Ray Conference 30 July - 06 August 2015. arXiv:1510.05223[astro.ph-HE]. 